\title{
MicroRNAs and Induced Pluripotent Stem Cells for Human Disease Mouse Modeling
}

\author{
Chingiz Underbayev, Siddha Kasar, Yao Yuan, and Elizabeth Raveche \\ Department of Pathology and Laboratory Medicine, University of Medicine and Dentistry of New Jersey, 185 South Orange Avenue, \\ MSB C512, Newark, NJ 07103, USA \\ Correspondence should be addressed to Elizabeth Raveche, raveches@umdnj.edu
}

Received 10 December 2011; Accepted 14 February 2012

Academic Editor: Monica Fedele

Copyright (๑) 2012 Chingiz Underbayev et al. This is an open access article distributed under the Creative Commons Attribution License, which permits unrestricted use, distribution, and reproduction in any medium, provided the original work is properly cited.

\begin{abstract}
Human disease animal models are absolutely invaluable tools for our understanding of mechanisms involved in both physiological and pathological processes. By studying various genetic abnormalities in these organisms we can get a better insight into potential candidate genes responsible for human disease development. To this point a mouse represents one of the most used and convenient species for human disease modeling. Hundreds if not thousands of inbred, congenic, and transgenic mouse models have been created and are now extensively utilized in the research labs worldwide. Importantly, pluripotent stem cells play a significant role in developing new genetically engineered mice with the desired human disease-like phenotype. Induced pluripotent stem (iPS) cells which represent reprogramming of somatic cells into pluripotent stem cells represent a significant advancement in research armament. The novel application of microRNA manipulation both in the generation of iPS cells and subsequent lineage-directed differentiation is discussed. Potential applications of induced pluripotent stem cell—a relatively new type of pluripotent stem cells_-for human disease modeling by employing human iPS cells derived from normal and diseased somatic cells and iPS cells derived from mouse models of human disease may lead to uncovering of disease mechanisms and novel therapies.
\end{abstract}

\section{Human Disease Mouse Models}

Model organisms such as fruit flies, zebrafish, and mice have provided great insights into gene function in humans because they are easy to grow and genetically manipulate in the laboratory setting. By evaluating different mutations in these organisms, one can identify candidate genes that lead to disease in humans and develop models to better understand human disease pathogenesis [1]. The mouse is an ideal model organism for human disease. Not only they are physiologically similar to humans, but a large genetic reservoir of potential models of human disease has been accumulated through the generation of radiation- or chemically induced mutant loci. Multiple technological advances have dramatically advanced our skills to create mouse models of human diseases. High-resolution genetic and physical linkage maps of the mouse genome have greatly facilitated the identification and cloning of mouse disease genes. Furthermore, transgenic approaches allowed us to ectopically express or make germline mutations in virtually any gene in the mouse genome by using homologous recombination in embryonic stem (ES) cells [2, 3]. Inbred, congenic and transgenic strains are widely used in current research labs as very valuable tools to investigate human diseases pathogenesis and develop new effective therapeutical strategies.

\section{Embryonic Stem (ES) and Induced Pluripotent Stem (iPS) Cells}

Pluripotency is the ability of a cell to give rise to progeny representing all types of cells in an organism [4]. Murine embryonic stem cells derived from inner cell mass (ICM) of the embryo exhibit two remarkable features in culture. First, under certain conditions, they can be propagated indefinitely as a stable self-renewing population where every cell undergoes symmetrical division. This immortalized phenotype allows ES cells to be cultured over extended periods of time. Upon differentiation, this feature is lost and progeny undergoes cellular aging (Hayflick limit) as has 


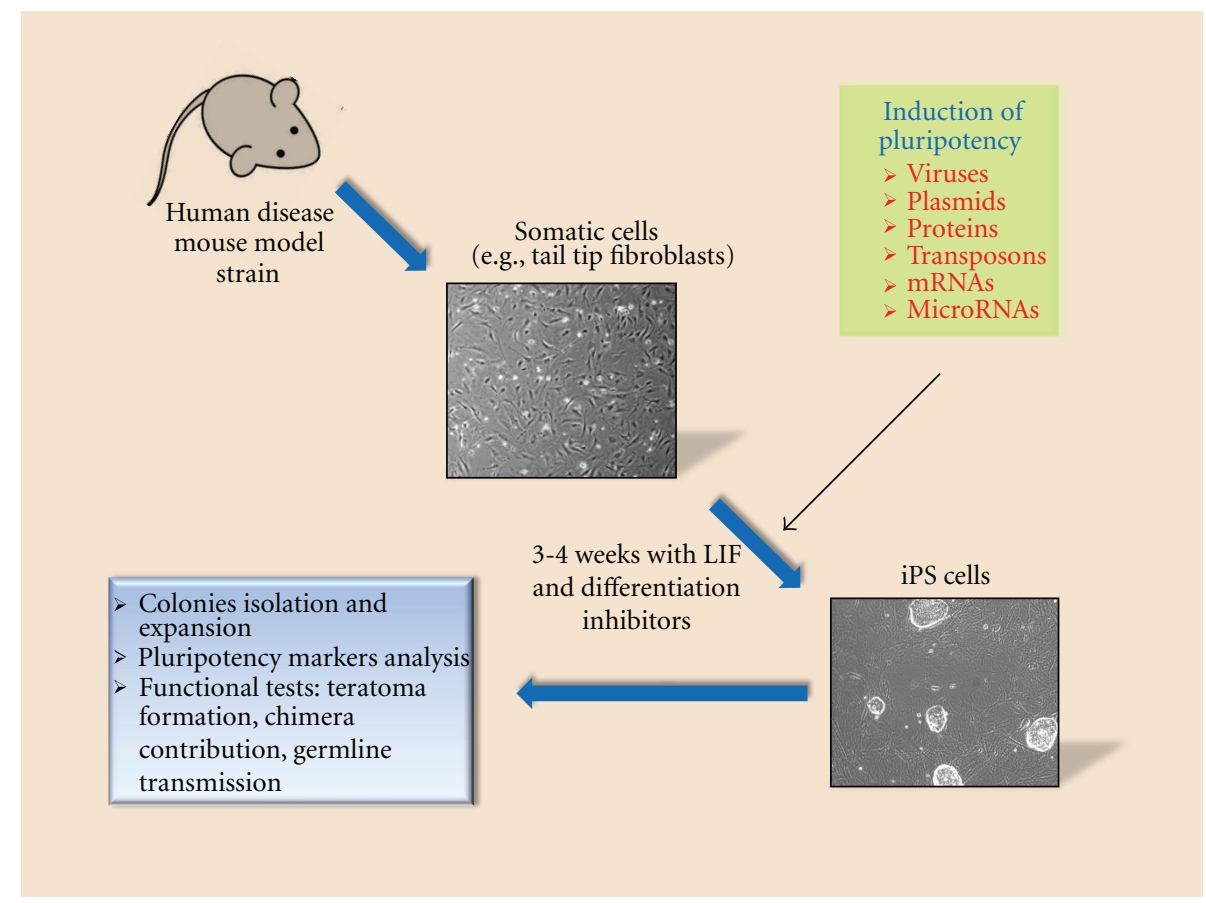

FIGURE 1: Generation of induced pluripotent stem cells from mouse model somatic cells.

been previously documented for all other nontransformed primary cells [5]. A second feature is that, during culture, ES cells retain their pluripotency and can differentiate into the same range of cell types as those seen in the embryo from ICM. The value of ES cells is partly due to their amenability to extensive gene manipulation. Homologous recombination between genomic and the exogenous DNA is a very inefficient and rare process, but it takes place in ES cells with relatively higher efficiency than it does in other cell types [4]. Gene targeting by homologous recombination in ES cells has improved our ability to study many biological processes [3]. Since ES cells contribute to all tissues upon injection into a recipient blastocyst, including the germline $[6,7]$ modification in an ES cell genome can be transmitted, by the breeding of ES cell/wild-type chimaeras, to generate mice containing the desired mutations in all cells. In this way mice with a variety of modifications such as null and point mutations, chromosomal rearrangements and large deletions have been generated. In addition, it is possible to target reporter genes under the control of specific promoters to study gene expression patterns in different cell types. Furthermore, the ability of ES cells to differentiate in vitro to many different mature somatic cell types, in combination with purification of the cell of interest by methods such as directed differentiation and lineage selection, opens up the opportunity to use these mature cell types for various basic and therapeutical applications [3]. Unfortunately, not every mouse model is permissive for true ES cells derivation. This makes it harder to investigate gene function and pathogenesis in those strains. With the advent of iPS technology this issue has been overcome.

In 2006, Takahashi and Yamanaka initially reported the direct reprogramming of murine embryonic fibroblasts (MEFs) to pluripotent stem cells by introducing four transcription factors [8]. Those factors, namely, Oct4, Sox2, $K l f 4$, and $c M y c$, that are important for self-renewal of embryonic stem cells (ESCs) have been shown to reprogram both mouse and human somatic cells into ESC-like pluripotent cells (Figure 1). Since then, a large number of laboratories have derived induced pluripotent stem cells from somatic cells, and many important advances have been made [9-15]. Most importantly these iPS cells have shown properties very similar to the ones of ES cells such as pluripotency markers expression, teratoma formation, chimeras contribution, and germline transmission. Moreover, the critical advantages of iPS cells over ES cells now seem to be obvious. First of all iPS cells are being generated from the autologous recipient thus obviating the graft-versus-host problem in transplantation settings. The second benefit pertains to the ethical concerns. Unlike in the past, one can now generate ES-like iPS cells from human skin fibroblasts or hair-follicle cells without the need to resort to the human ES cell lines and potentially (in the future) apply them to the therapeutic and/or basic science approaches [13].

\section{Making Use of iPS Cells for Human Disease Modeling}

For the human disease animal modeling iPS cell technology opened the way to even wider spectrum of available mouse model strains. In 2009, Zhao et al. reported the generation of all-iPS-derived viable, fertile live-born progeny by tetraploid complementation [16] which further proved them to be useful for the development of transgenic mice strains with desired gene defects homologous to those seen in human pathology. 


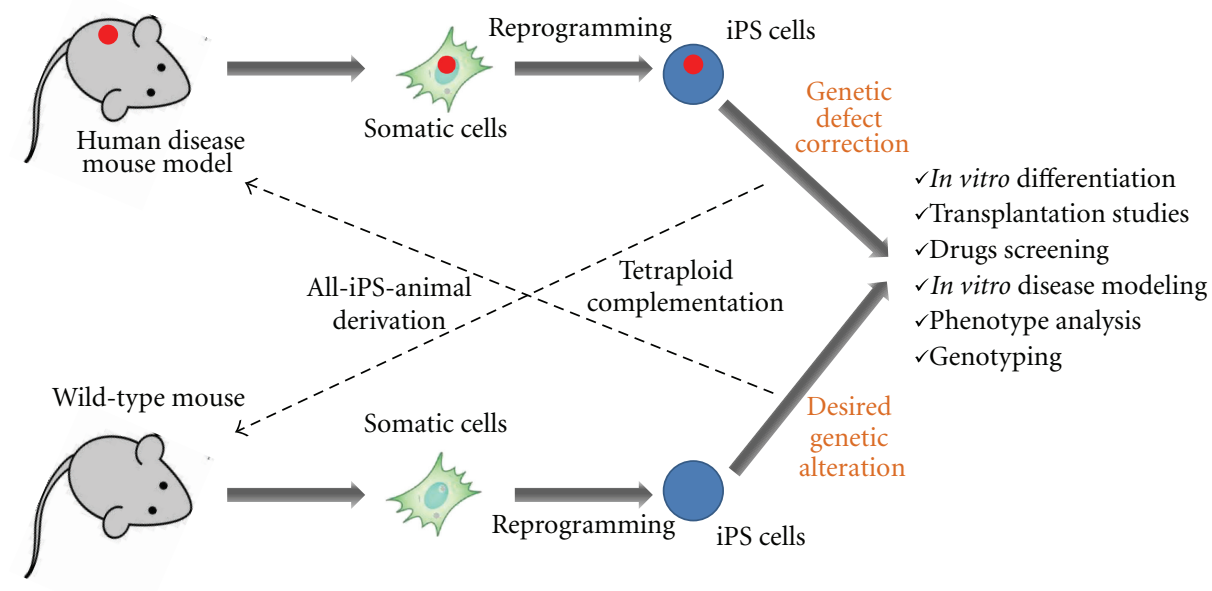

FIGURE 2: Mouse induced pluripotent stem cells applications for human disease mouse modeling.

At present, with this valuable tool in hand one can take literally any human disease mouse strain somatic cells (e.g., tail tip fibroblasts) and induce pluripotent stem cells from them. These disease-specific iPS cells can be further used to explore given disease mechanisms both in vitro and in vivo. (Figure 2). For example, human chronic lymphocytic leukemia (CLL) (CD5+ B-cell malignancy) mouse modelNew Zealand Black mouse-exhibits a defect in the miR15a/16-1 gene on chromosome 14 which results in decreased levels of these microRNAs, which is also seen in more than $50 \%$ of CLL patients [17]. Unfortunately, this mouse strain is refractory to true ES cells derivation which makes it difficult to study the role of this microRNA gene defect in B-cell development both in vitro and in vivo. In our lab, we were able to successfully generate NZB iPS cells from spleen stromal cells. Now they can be used as subjects for gene targeting (correcting miR-15a/16-1 mutation and deletion) followed by in vitro differentiation towards B-lineage. This would help find out what role this particular gene defect plays in B-cell lymphogenesis and how its correction might alleviate malignant clonal expansion. Furthermore, NZB iPS cells with corrected miR-15a defect could be differentiated into hematopoietic stem cells (HSCs) followed by their adoptive transfer into appropriate recipients in order to observe the effect of gene correction on CLL development in vivo.

Another way to utilize iPS cells to study human diseases in animal models is xenograft transplantation assay. In this case iPS cells would be generated from patient's somatic cells (Figure 3), differentiated into desired type of cells (e.g., HSC), and transplanted into immunodeficient murine recipients. In a recent report, Yao et al. [18] have demonstrated a generation of human iPS cells with zinc-finger nuclease, mediated disruption of CCR5 locus which is known to be a coreceptor for HIV entry. These patient-specific iPS cells can now be differentiated into HSC and transplanted into animal recipients to study the role of CCR5 in HIV infection development in vivo. In another work, Lee et al. have used human iPS-derived neural stem cells (NSCs) in a mouse intracranial human glioma xenograft model [19]. In this case, iPS-derived NSCs have been used as cellular vehicles for targeted anticancer gene therapy since they will home to the brain. As a proof of principle, Hanna and colleagues have taken advantage of autologous iPS cells derived from humanized mouse model of sickle cells anemia to correct human sickle hemoglobin allele by gene-specific targeting followed by their differentiation into hematopoietic stem cells and transplantation into irradiated recipients [20]. It has been shown that mice could be rescued from disease progression after transplantation. This work has underlined the benefits of iPS technology for the combined gene and cell therapy approach to study human disease in animal models. It is needless to say that currently various labs worldwide use patient-specific iPS cells for animal modeling both in vitro and in vivo. Such pathological conditions as Huntington disease, amyotrophic lateral sclerosis, spinal muscular dystrophy, Gaucher disease type III, Down syndrome, type 1 diabetes, Parkinson's disease, $\beta$-thalassemia, and hepatic failure have been investigated using iPS cells generation [20-29].

\section{MicroRNAs}

MicroRNAs (miRs) are small noncoding RNAs which are known to be critical for the expression control of more than a third of all protein coding genes [30] by means of binding to the $3^{\prime}$ untranslated region (UTR) of target mRNAs via an imperfect match to repress their translation and/or stability [31]. They have been implicated in the regulation of many biological processes, including the stem cells self-renewal and pluripotency [32-34]. MiRNAs are generated from precursor transcripts - primary miRNAs (pri-miRNAs) — that are first processed in the nucleus into an intermediate pre-miRNAs by the complex of enzymes containing Drosha and DGCR8 proteins [35-37]. The pre-miRNAs are then transported by the exportin 5-RanGTP shuttle into the cytoplasm, in which they are further processed by Dicer, into mature miRNAs [38]. In ES cells a set of microRNAs (including miR-302 and miR-17-92 clusters) closely interfere with the 


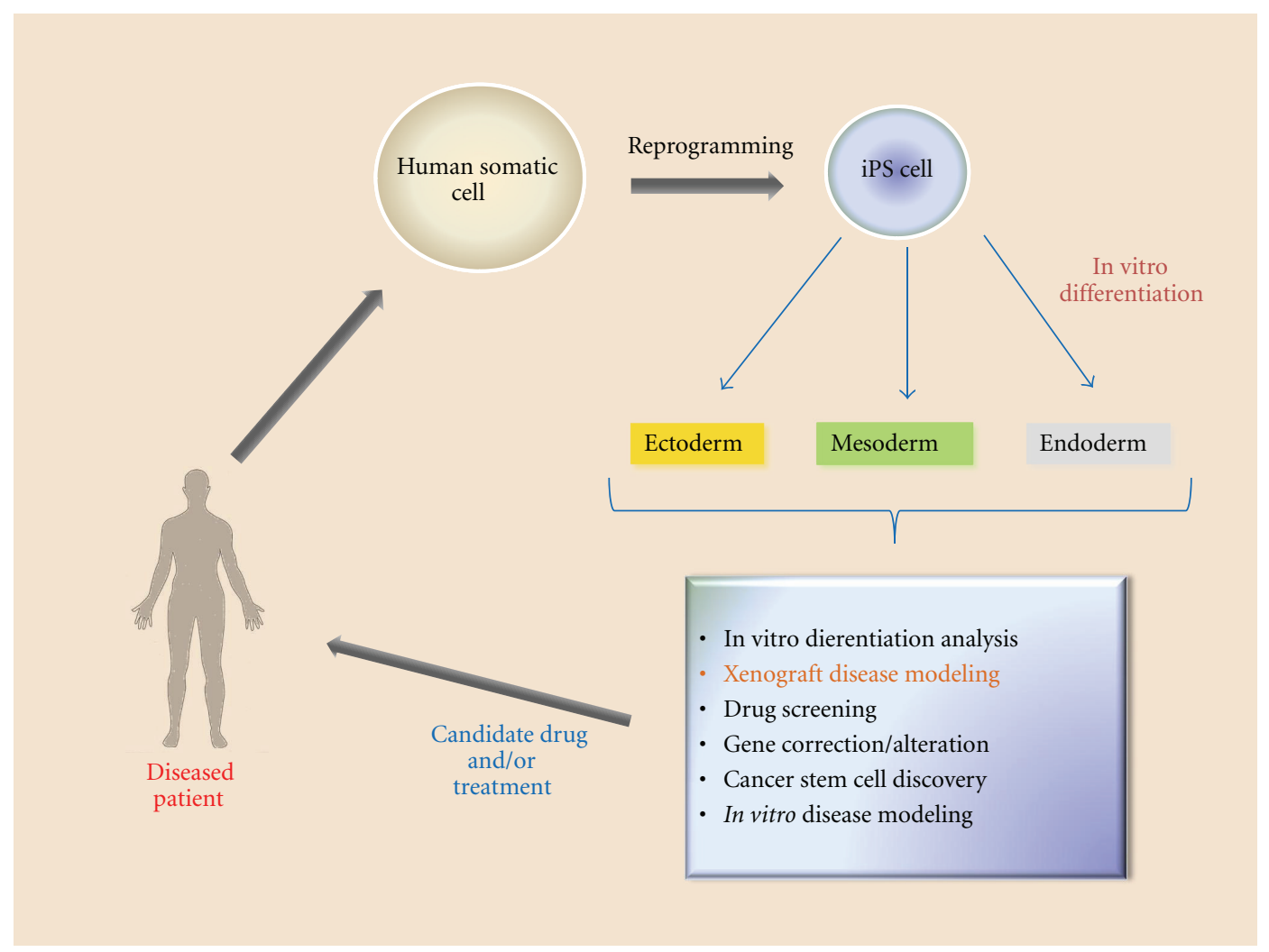

FIGURE 3: Human induced pluripotent stem cells applications for human disease mouse models.

key pluripotency factors such as Oct4, Sox2, and Nanog $[39,40]$ thereby preventing them from differentiation and controlling their proper self-renewal potential [41]. Xu et al. have demonstrated miR-145 to control the expression of Oct4, Sox2, and Klf4 and repress self-renewal of human ES cells [42]. On the other hand, c-Myc has been reported to repress miRNAs such as miR-21, let-7a, and miR-29a during reprogramming [43]. Tissue-specific miRNAs often play important roles in normal tissues and organ formation [44, 45]. More importantly for the current review, microRNAs proved to be effective tools for the iPS generation. In particular, inhibition of miR-21, let-7a, or mir-29a has been shown to enhance the reprogramming efficiency [43]. Alternatively, overexpression of the miR302/367 cluster has been shown to rapidly and efficiently reprogram both mouse and human somatic cells to iPS state without any exogenous transcription factors delivery through Oct4 gene expression activation and the suppression of Hdac2 [46]. MicroRNA gene expression profiling in human ES cells revealed specific miR-signatures of elevated expression of miR-302 cluster, miR-200 family members as well as miR-520 cluster [47]. This might imply the possibility of them to be used as tools to increase the efficiency of iPS generation without any exogenous interventions into the genomic DNA of the host cells and serve as additional iPS quality control markers. Conversely, as the regulators of gene expression microRNAs could be used to drive patient-specific iPS cells down the specific cells lineage in vitro in order to produce the required cell type to be studied [48].
Another promise that microRNAs are holding is the development of microRNA-based gene targeting for the temporal gene-of-interest silencing [49]. For instance, aberrant expression of Pax5 (also known as BSAP), a critical regulator of B-cell development, is known to correlate with aggressive subsets of B-cell non-Hodgkin lymphoma [50]. It has been previously shown that overexpression of miR-15a/16 reduces endogenous c-Myb levels and compromises Pax5 function [51]. Now one can produce iPS cells from Pax5-affected non-Hodgkin lymphoma patient and apply in vitro B-cell differentiation protocol along with miR-15a/16-1 delivery to evaluate lymphomagenesis in the mouse xenograft model. Potentially, the similar approach could be employed for the discovery of leukemia (or more commonly cancer) stem cells [52]. Finally, miRs can be used as biomarkers of human disease progression in mouse model settings. Overall diagram of microRNA application for mouse modeling is shown in Figure 4.

\section{Conclusion}

The importance of disease mouse models and their impact on medical research is hard to overestimate. Therefore the value of animal modeling is very critical for our understanding of human disease and development of new effective approaches to therapy. Induced pluripotent stem cells hold a great promise for both basic and applied science and open the road for many more opportunities for human disease research. Coupled with the use of fine-tune regulators 


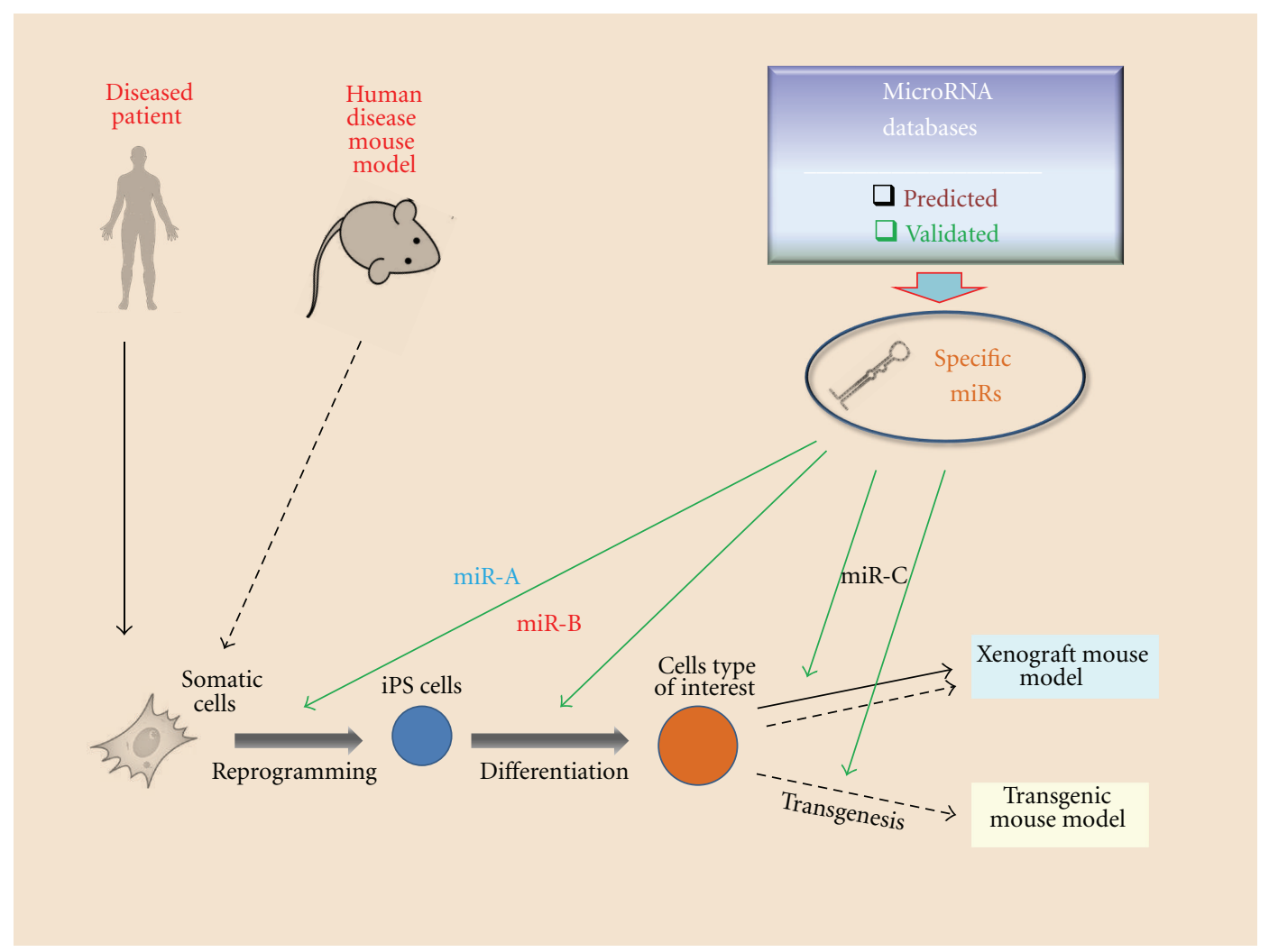

FIGURE 4: MicroRNA applications for iPS-mediated human disease mouse models.

of gene expression, microRNAs, and mouse modeling they have a promising potential for subsequent discoveries and new therapies development in the complex field of human pathology.

\section{References}

[1] N. L. Washington, M. A. Haendel, C. J. Mungall, M. Ashburner, M. Westerfield, and S. E. Lewis, "Linking human diseases to animal models using ontology-based phenotype annotation," PLoS Biology, vol. 7, no. 11, Article ID e1000247, 2009.

[2] M. A. Bedell, N. A. Jenkins, and N. G. Copeland, "Mouse models of human disease. Part I: techniques and resources for genetic analysis in mice," Genes and Development, vol. 11, no. 1, pp. 1-10, 1997.

[3] L. Hook, C. O’Brien, and T. Allsopp, "ES cell technology: an introduction to genetic manipulation, differentiation and therapeutic cloning," Advanced Drug Delivery Reviews, vol. 57, no. 13, pp. 1904-1917, 2005.

[4] H. Niwa, "Mouse ES cell culture system as a model of development," Development Growth and Differentiation, vol. 52, no. 3, pp. 275-283, 2010.

[5] S. Ohtsuka and S. Dalton, "Molecular and biological properties of pluripotent embryonic stem cells," Gene Therapy, vol. 15, no. 2, pp. 74-81, 2008.

[6] A. Bradley, M. Evans, M. H. Kaufman, and E. Robertson, "Formation of germ-line chimaeras from embryo-derived teratocarcinoma cell lines," Nature, vol. 309, no. 5965, pp. 255256,1984 .
[7] E. Robertson, A. Bradley, M. Kuehn, and M. Evans, "Germline transmission of genes introduced into cultured pluripotential cells by retroviral vector," Nature, vol. 323, no. 6087, pp. 445-448, 1986.

[8] K. Takahashi and S. Yamanaka, "Induction of pluripotent stem cells from mouse embryonic and adult fibroblast cultures by defined factors," Cell, vol. 126, no. 4, pp. 663-676, 2006.

[9] K. Okita, M. Nakagawa, H. Hyenjong, T. Ichisaka, and S. Yamanaka, "Generation of mouse induced pluripotent stem cells without viral vectors," Science, vol. 322, no. 5903, pp. 949953, 2008.

[10] M. Stadtfeld, M. Nagaya, J. Utikal, G. Weir, and K. Hochedlinger, "Induced pluripotent stem cells generated without viral integration," Science, vol. 322, no. 5903, pp. 945949, 2008.

[11] T. Seki, S. Yuasa, M. Oda et al., "Generation of induced pluripotent stem cells from human terminally differentiated circulating T cells," Cell stem cell, vol. 7, no. 1, pp. 11-14, 2010.

[12] M. Wernig, A. Meissner, R. Foreman et al., "In vitro reprogramming of fibroblasts into a pluripotent ES-cell-like state," Nature, vol. 448, no. 7151, pp. 318-324, 2007.

[13] J. Zou, M. L. Maeder, P. Mali et al., "Gene Targeting of a Disease-Related Gene in Human Induced Pluripotent Stem and Embryonic Stem Cells," Cell Stem Cell, vol. 5, no. 1, pp. 97-110, 2009.

[14] M. I. Lai, W. Y. Wendy-Yeo, R. Ramasamy et al., "Advancements in reprogramming strategies for the generation of induced pluripotent stem cells," Journal of Assisted Reproduction and Genetics, vol. 28, no. 4, pp. 291-301, 2011.

[15] K. Takahashi, "Direct reprogramming 101," Development Growth and Differentiation, vol. 52, no. 3, pp. 319-333, 2010. 
[16] X. Y. Zhao, W. Li, Z. Lv et al., "IPS cells produce viable mice through tetraploid complementation," Nature, vol. 461, no. 7260, pp. 86-90, 2009.

[17] E. Salerno, B. J. Scaglione, F. D. Coffman et al., "Correcting miR-15a/16 genetic defect in New Zealand Black mouse model of CLL enhances drug sensitivity," Molecular Cancer Therapeutics, vol. 8, no. 9, pp. 2684-2692, 2009.

[18] Y. Yao, B. Nashun, T. Zhou et al., "Generation of CD34+ cells from CCR5-disrupted human embryonic and induced pluripotent stem cells," Human Gene Therapy, vol. 23, no. 2, pp. 238-242, 2012.

[19] E. X. Lee, D. H. Lam, C. Wu et al., "Glioma gene therapy using induced pluripotent stem cell derived neural stem cells," Molecular Pharmaceutics, vol. 8, no. 5, pp. 1515-1524, 2011.

[20] J. Hanna, M. Wernig, S. Markoulaki et al., "Treatment of sickle cell anemia mouse model with iPS cells generated from autologous skin," Science, vol. 318, no. 5858, pp. 1920-1923, 2007.

[21] J. T. Dimos, K. T. Rodolfa, K. K. Niakan et al., "Induced pluripotent stem cells generated from patients with ALS can be differentiated into motor neurons," Science, vol. 321, no. 5893, pp. 1218-1221, 2008.

[22] A. D. Ebert, J. Yu, F. F. Rose et al., "Induced pluripotent stem cells from a spinal muscular atrophy patient," Nature, vol. 457, no. 7227, pp. 277-280, 2009.

[23] I. H. Park, N. Arora, H. Huo et al., "Disease-specific induced pluripotent stem cells," Cell, vol. 134, no. 5, pp. 877-886, 2008.

[24] F. Soldner, D. Hockemeyer, C. Beard et al., "Parkinson's disease patient-derived induced pluripotent stem cells free of viral reprogramming factors," Cell, vol. 136, no. 5, pp. 964-977, 2009.

[25] L. Ye, J. C. Chang, C. Lin, X. Sun, J. Yu, and Y. W. Kan, "Induced pluripotent stem cells offer new approach to therapy in thalassemia and sickle cell anemia and option in prenatal diagnosis in genetic diseases," Proceedings of the National Academy of Sciences of the United States of America, vol. 106, no. 24, pp. 9826-9830, 2009.

[26] M. Wernig, J. P. Zhao, J. Pruszak et al., "Neurons derived from reprogrammed fibroblasts functionally integrate into the fetal brain and improve symptoms of rats with Parkinson's disease," Proceedings of the National Academy of Sciences of the United States of America, vol. 105, no. 15, pp. 5856-5861, 2008.

[27] Q. Zhou, J. Brown, A. Kanarek, J. Rajagopal, and D. A. Melton, "In vivo reprogramming of adult pancreatic exocrine cells to $\beta$-cells," Nature, vol. 455, no. 7213, pp. 627-632, 2008.

[28] I. S. Behbahan, Y. Duan, A. Lam et al., "New approaches in the differentiation of human embryonic stem cells and induced pluripotent stem cells toward hepatocytes," Stem Cell Reviews and Reports, vol. 7, no. 3, pp. 748-759, 2011.

[29] S. Asgari, M. Moslem, K. Bagheri-Lankarani, B. Pournasr, M. Miryounesi, and H. Baharvand, "Differentiation and transplantation of human induced pluripotent stem cellderived hepatocyte-like cells," Stem Cell Reviews and Reports. In press.

[30] S. K. Mallanna and A. Rizzino, "Emerging roles of microRNAs in the control of embryonic stem cells and the generation of induced pluripotent stem cells," Developmental Biology, vol. 344, no. 1, pp. 16-25, 2010.

[31] T. M. Rana, "Illuminating the silence: understanding the structure and function of small RNAs," Nature Reviews Molecular Cell Biology, vol. 8, no. 1, pp. 23-36, 2007.

[32] C. Melton, R. L. Judson, and R. Blelloch, "Opposing microRNA families regulate self-renewal in mouse embryonic stem cells," Nature, vol. 463, no. 7281, pp. 621-626, 2010.
[33] Y. Tay, J. Zhang, A. M. Thomson, B. Lim, and I. Rigoutsos, "MicroRNAs to Nanog, Oct4 and Sox 2 coding regions modulate embryonic stem cell differentiation," Nature, vol. 455, no. 7216, pp. 1124-1128, 2008.

[34] Y. M. S. Tay, W. L. Tam, Y. S. Ang et al., "MicroRNA-134 modulates the differentiation of mouse embryonic stem cells, where it causes post-transcriptional attenuation of Nanog and LRH1," Stem Cells, vol. 26, no. 1, pp. 17-29, 2008.

[35] Y. Lee, C. Ahn, J. Han et al., "The nuclear RNase III Drosha initiates microRNA processing," Nature, vol. 425, no. 6956, pp. 415-419, 2003.

[36] Y. Lee, K. Jeon, J. T. Lee, S. Kim, and V. N. Kim, "MicroRNA maturation: stepwise processing and subcellular localization," EMBO Journal, vol. 21, no. 17, pp. 4663-4670, 2002.

[37] Y. Zeng and B. R. Cullen, "Sequence requirements for micro RNA processing and function in human cells," RNA, vol. 9, no. 1, pp. 112-123, 2003.

[38] V. K. Gangaraju and H. Lin, "MicroRNAs: key regulators of stem cells," Nature Reviews Molecular Cell Biology, vol. 10, no. 2, pp. 116-125, 2009.

[39] A. Marson, S. S. Levine, M. F. Cole et al., "Connecting microRNA genes to the core transcriptional regulatory circuitry of embryonic stem cells," Cell, vol. 134, no. 3, pp. 521533, 2008.

[40] K. D. Wilson, S. Venkatasubrahmanyam, F. Jia, N. Sun, A. J. Butte, and J. C. Wu, "MicroRNA profiling of human-induced pluripotent stem cells," Stem Cells and Development, vol. 18, no. 5, pp. 749-757, 2009.

[41] Y. Wang, R. Medvid, C. Melton, R. Jaenisch, and R. Blelloch, "DGCR8 is essential for microRNA biogenesis and silencing of embryonic stem cell self-renewal," Nature Genetics, vol. 39, no. 3, pp. 380-385, 2007.

[42] N. Xu, T. Papagiannakopoulos, G. Pan, J. A. Thomson, and K. S. Kosik, "MicroRNA-145 regulates OCT4, SOX2, and KLF4 and represses pluripotency in human embryonic stem cells," Cell, vol. 137, no. 4, pp. 647-658, 2009.

[43] C. S. Yang, Z. Li, and T. M. Rana, "microRNAs modulate iPS cell generation," RNA, vol. 17, no. 8, pp. 1451-1460, 2011.

[44] K. N. Ivey, A. Muth, J. Arnold et al., "MicroRNA regulation of cell lineages in mouse and human embryonic stem cells," Cell Stem Cell, vol. 2, no. 3, pp. 219-229, 2008.

[45] Y. Zhao, E. Samal, and D. Srivastava, "Serum response factor regulates a muscle-specific microRNA that targets Hand2 during cardiogenesis," Nature, vol. 436, no. 7048, pp. 214-220, 2005.

[46] F. Anokye-Danso, C. M. Trivedi, D. Juhr et al., "Highly efficient miRNA-mediated reprogramming of mouse and human somatic cells to pluripotency," Cell Stem Cell, vol. 8, no. 4, pp. 376-388, 2011.

[47] J. Ren, P. Jin, E. Wang, F. M. Marincola, and D. F. Stroncek, "MicroRNA and gene expression patterns in the differentiation of human embryonic stem cells," Journal of Translational Medicine, vol. 7, article 20, 2009.

[48] L. R. Saunders et al., "miRNAs regulate SIRT1 expression during mouse embryonic stem cell differentiation and in adult mouse tissues," Aging, vol. 2, no. 7, pp. 415-431, 2010.

[49] C. C. Lan, I. U. S. Leong, D. Lai, and D. R. Love, "Disease modeling by gene targeting using MicroRNAs," Methods in Cell Biology, vol. 105, pp. 419-436, 2011.

[50] L. Krenacs, A. W. Himmelmann, L. Quintanilla-Martinez et al., "Transcription factor B-cell-specific activator protein (BSAP) is differentially expressed in B cells and in subsets of B-cell lymphomas," Blood, vol. 92, no. 4, pp. 1308-1316, 1998. 
[51] E. Y. Chung, M. Dews, D. Cozma et al., "c-Myb oncoprotein is an essential target of the dleu2 tumor suppressor microRNA cluster," Cancer Biology and Therapy, vol. 7, no. 11, pp. 17581764, 2008.

[52] P. H. Gunaratne, "Embryonic stem cell MicroRNAs: defining factors in induced pluripotent (iPS) and cancer (CSC) stem cells?" Current Stem Cell Research and Therapy, vol. 4, no. 3, pp. 168-177, 2009. 


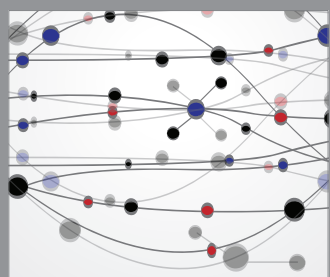

The Scientific World Journal
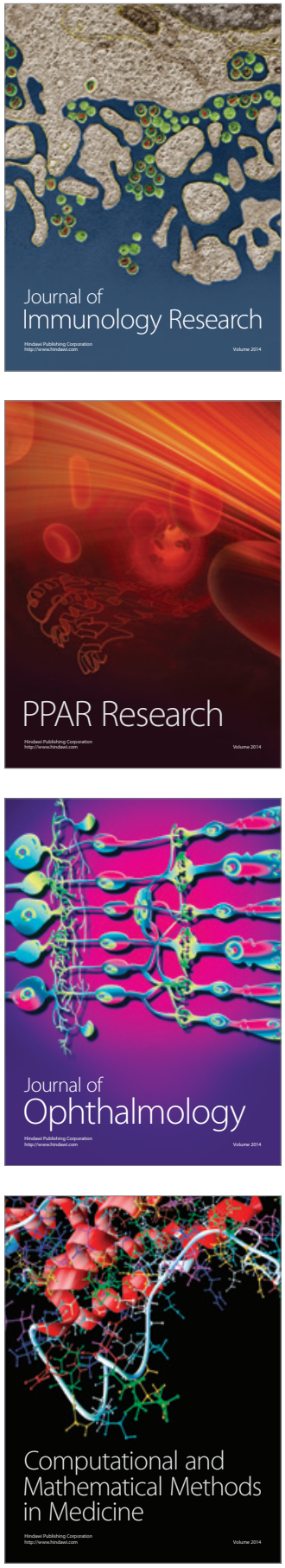

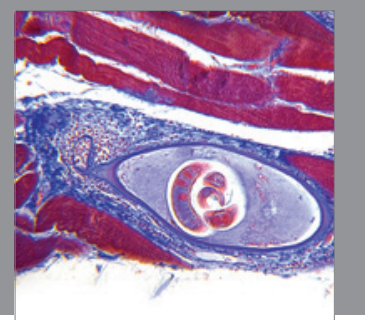

Gastroenterology

Research and Practice
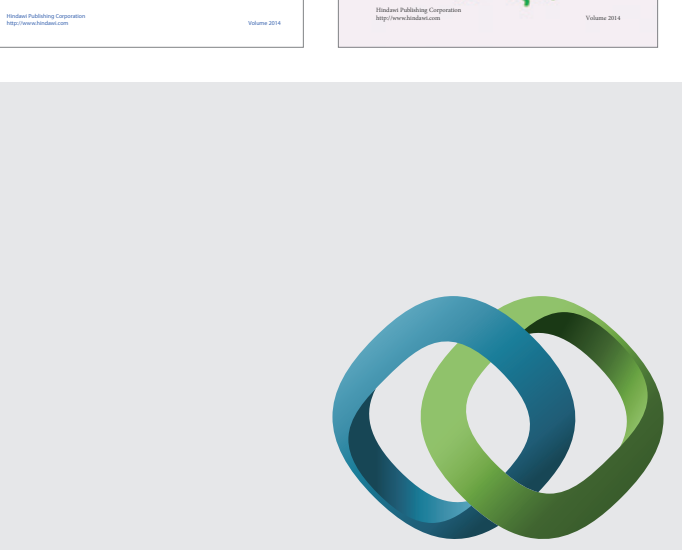

\section{Hindawi}

Submit your manuscripts at

http://www.hindawi.com
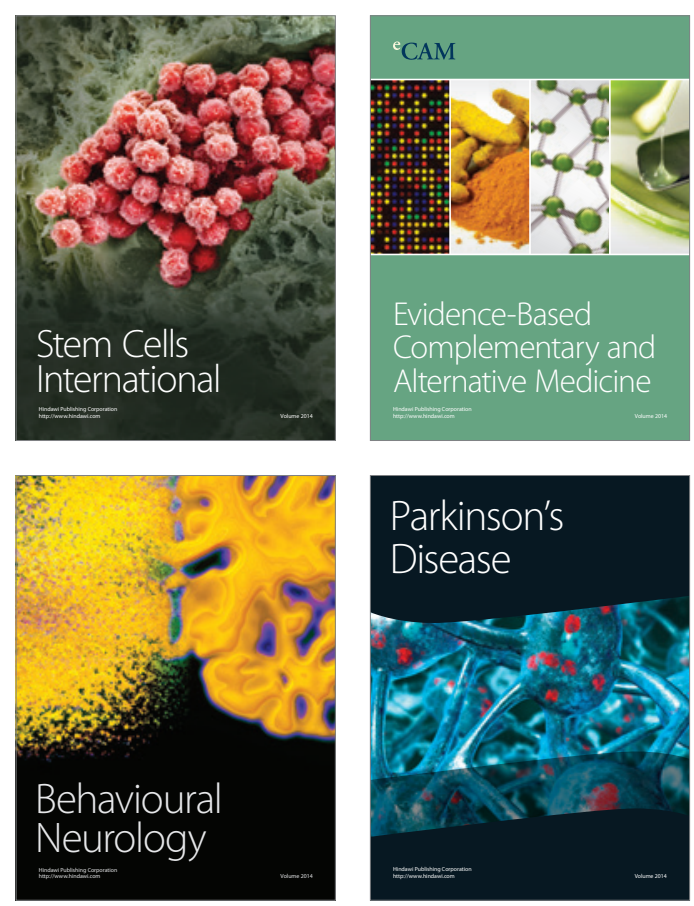

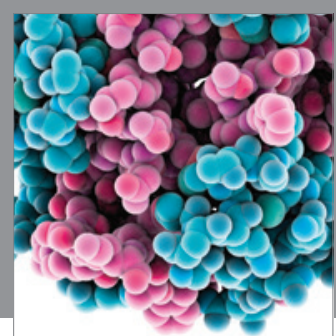

Journal of
Diabetes Research

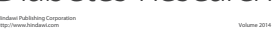

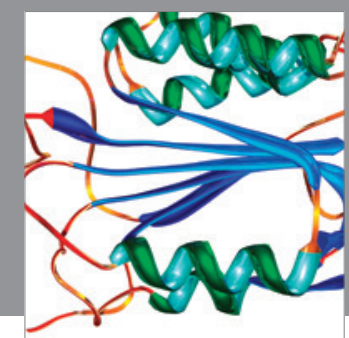

Disease Markers
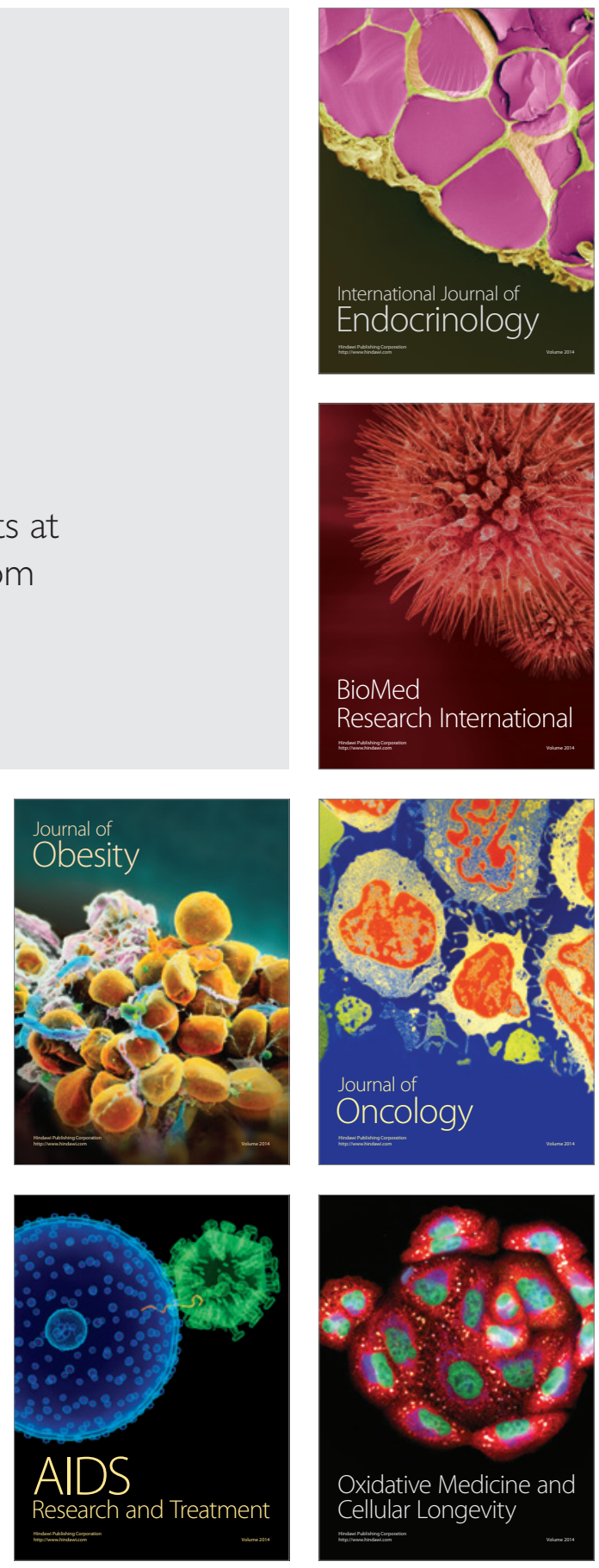